\title{
L'inclusion scolaire pour gérer la diversité: des aspects théoriques aux pratiques dites efficaces
}

\section{Nadia Rousseau, Geneviève Bergeron et Raymond Vienneau}

Plusieurs pays déploient des efforts considérables dans le but de développer des systèmes scolaires plus inclusifs. Ces démarches ont ouvert plusieurs chantiers de recherche au cours des dernières décennies, dont l'un davantage centré sur le développement d'un modèle théorique de l'inclusion scolaire et un autre cherchant à évaluer les effets de différentes pratiques, plus ou moins inclusives. Cet article propose une synthèse des recherches menées dans chacun de ces deux champs de recherche, relativement récents. Nous aborderons ainsi différents aspects conceptuels: définitions, postulats d'une école dite inclusive, caractéristiques d'une pédagogie de l'inclusion et conditions essentielles à sa mise en ouvre. Dans la dernière section, on se penchera sur les effets de l'inclusion scolaire sur l'apprentissage et le développement social des élèves avant de conclure sur un troisième champ de recherche émergent: les pratiques efficaces.

Qu'on la désigne sous son appellation traditionnelle d' «intégration-mainstreaming» (Doré, Wagner, Brunet \& Bélanger, 1998) ou sous sa manifestation la plus récente et la plus ambitieuse de pédagogie de l'inclusion (Rousseau \& Bélanger, 2004), l'inclusion scolaire des élèves avec handicaps ou en difficulté d'adaptation ou d'apprentissage est un mouvement dont les fondements sociologiques et philosophiques sont désormais bien établis (Vienneau, 2002). L'inclusion scolaire s'appuie, entre autres arguments, sur des principes d'égalité des droits éducatifs et d'un accès universel à l'institution qui «joue un rôle fondamental dans l'apprentissage des règles de la vie en société et dans le processus d'intégration sociale des individus: l'École» (Ducharme, 2008, p. 5). De plus, dans un esprit d'équité sociopédagogique (Lafortune, 2006), un certain consensus semble aujourd'hui émerger autour de la légitimité de la diversité en éducation (Prud'homme, Vienneau, Ramel \& Rousseau, 2011), et ce, quelle que soit l'étendue des différences susceptibles d'être rencontrées au sein des élèves composant le groupe-classe (Vienneau, 2006). Ainsi, le passage d'un modèle d'école centré sur l'homogénéité à celui d'une école centrée sur l'hétérogénéité s'inscrit dans une perspective de dénormalisation (AuCoin \& Vienneau, 2010) menant à l'implantation de pratiques inclusives, voire d'une pédagogie qu'on 
pourrait même qualifier d'universelle (Bergeron, Rousseau \& Leclerc, 2011; Rousseau, 2010a).

L'inclusion scolaire continue d'alimenter de nombreuses questions de recherche et de développement en éducation, dont les problématiques associées à la conceptualisation même du phénomène, à ses postulats, à ses aspects opérationnels et à ses conditions de mise en ouvre. On pourrait faire correspondre ces diverses problématiques au développement d'un modèle théorique de l'inclusion scolaire et de son corollaire pédagogique: la pédagogie de l'inclusion (Rousseau, 2010b; Rousseau \& Bélanger, 2004).

Ces problématiques d'ordres conceptuel et théorique, sont évidemment loin d'être les seules à avoir été étudiées et documentées au cours des dernières décennies. Associés de près aux aspects opérationnels de l'inclusion scolaire, d'autres chercheurs étudient ou proposent différents modèles de services pour favoriser l'intégration scolaire (p. ex., Trépanier \& Paré, 2010) ou pour faciliter l'intégration pédagogique dans un contexte inclusif (p. ex., Benoit \& Angelucci, 2011). Parmi d'autres recherches, mentionnons la problématique des attitudes envers les élèves avec handicaps ou en difficulté (p. ex., Bélanger, 2010); les études visant à décrire le rôle des divers intervenants scolaires (p. ex., Moreau, 2010), incluant le rôle de leadership exercé par la direction d'école (Parent, 2010); le développement et l'évaluation de dispositifs de soutien à l'inclusion scolaire (p. ex., Bonvin, 2011); les défis de la formation initiale (p. ex., Garbo \& Albanese, 2006), de la formation continue (p. ex., Prud'homme, 2010) et de l'accompagnement des intervenants scolaires (Ouellet, Caya \& Tremblay, 2011); les impacts de l'intégration et de l'inclusion scolaires chez les enseignants (p. ex., Ramel, 2010); les aspects légaux, les droits éducatifs et l'analyse des politiques scolaires (AuCoin, Goguen \& Vienneau, 2011).

Sans être exhaustive, cette liste illustre néanmoins l'étendue et la diversité des thèmes de recherche associés à l'inclusion scolaire. Étant donné les limites de cet article, nous avons choisi de nous concentrer sur les aspects conceptuels et théoriques du phénomène (fondements théoriques), qui regroupent certaines des thématiques citées ci-dessus, auxquels nous avons choisi d'associer un axe de recherche particulièrement fructueux et déterminant dans le développement de politiques inclusives, soit celui de l'étude des effets de l'inclusion scolaire sur l'apprentissage et le développement social des élèves (fondements empiriques).

\section{Regard sur le concept de L'inclusion ${ }^{1}$}

Souvent utilisé comme descripteur général, le mot «inclusion» est un terme polysémique qui s'associe à une importante terminologie: inclusion scolaire, pédagogie de l'inclusion, éducation inclusive, école inclusive. Stainback et Stainback (1990), précurseurs du mouvement inclusif qui s'est développé graduellement aux États-Unis à partir des années 1990, font partie des premiers à utiliser le 
concept d'inclusion en contexte éducatif (Thomazet, 2008). Aujourd'hui, les termes «éducation inclusive» ou "école inclusive» sont fréquemment employés comme synonymes de l'inclusion scolaire. Cependant, l'éducation inclusive apparaît comme un concept plus englobant se référant à différentes initiatives dont l'objectif est de lutter contre l'exclusion sociale. Elle renvoie communément au droit de tous les jeunes à recevoir une éducation de qualité, et ce, sans égard aux différences qu'ils peuvent manifester (Armstrong, 2006; Moran, 2007; UNESCO, 1994). En outre, l'éducation inclusive est étroitement reliée aux objectifs d'éducation pour tous (Education for All) promus par l'UNESCO depuis plus de 20 ans. Le vocable «pédagogie de l'inclusion» est également utilisé par certains chercheurs et certaines chercheuses (Rousseau, 2010b; Vienneau, 2002). Même si quelques-uns (AuCoin \& Vienneau, 2010; Vienneau, 2006) rappellent que l'inclusion scolaire, dans les faits, «n'est pas toujours synonyme de pédagogie de l'inclusion» (Vienneau, 2006, p. 17), les deux concepts sont souvent utilisés comme synonymes et sont généralement concentrés sur les mêmes idées (Bergeron, en préparation).

Pour plusieurs raisons, il s'avère complexe de circonscrire avec justesse chacun de ces concepts. D'abord, les définitions peuvent comporter certaines variations selon les chercheurs et les chercheuses et les écrits consultés. De surcroît, plusieurs de ces écrits privilégient l'emploi d'un terme plutôt qu'un autre sans toutefois préciser les raisons qui sous-tendent leur choix ou encore sans définir explicitement les dénominations retenues (Bergeron, en préparation). Ajoutant à la confusion, d'autres utilisent plusieurs de ces concepts de manière synonymique dans un même écrit. En somme, force est de constater que différents termes sont utilisés pour parler de différents phénomènes (Ainscow, Farrell \& Tweddle, 2000; Beauregard \& Trépanier, 2010; Boutin \& Bessette, 2009; Haug, 2003; Vienneau, 2004). Indépendamment du vocable privilégié, il importe également de reconnaitre que toute initiative inclusive s'inscrit dans un contexte éducatif particulier et peut donc prendre un sens quelque peu différent. En effet, loin de se concevoir comme une approche astreignante, ces initiatives peuvent être mises en œuvre à différents niveaux, viser des objectifs différents, se fonder sur des motifs différents, toucher différentes catégories de besoins éducatifs particuliers ou encore fournir des services dans des contextes différents (Peters, 2007). Comme le soulignent Rouse et Florian (1996), les façons de faire sont et doivent être spécifiques à chaque milieu et contexte éducatifs particuliers.

Il n'y a donc pas de modèle unique pour décrire l'inclusion scolaire ou l'école inclusive (Mitchell, 2005). Toutefois, on s'entend généralement pour préciser ce qu'elle n'est pas et pour reconnaître une opposition paradigmatique entre l'intégration scolaire ou l'intégration-mainstreaming, comme la désignent Doré et ses collaborateurs (Doré, Wagner, Brunet $\&$ Bélanger, 1998), et l'inclusion scolaire (Vienneau, 2006). Rappelons brièvement à ce sujet que l'intégration scolaire, amorcée au courant des années 1970, se contentait de permettre le placement de l'élève en difficulté dans l'environnement scolaire le moins restrictif possible. 
L'école pouvait donc continuer d'offrir tout un continuum de services, allant du plus ségrégué (p. ex., l'école spécialisée) au plus intégré (l'intégration pédagogique à temps plein dans une classe ordinaire). Il en va tout autrement avec le paradigme de l'inclusion scolaire, qui abolit toute forme de rejet (zero reject philosophy) et préconise, dans sa version la plus radicale (full inclusion), un seul placement pour tous les élèves, quels que soient leurs handicaps ou leurs particularités de fonctionnement.

$\mathrm{Au}$ sens large, plusieurs chercheurs et chercheuses conçoivent en effet l'inclusion scolaire comme un phénomène étroitement lié à l'adoption de valeurs sociales et éducatives de justice et d'équité (Moran, 2007; UNESCO, 1994). Plus spécifiquement, nombreux sont les acteurs, les actrices et les chercheurs et chercheuses du champ éducatif qui choisissent d'inscrire le développement d'écoles inclusives dans une vision transformative de la société (Hick, Kershner $\&$ Farrel, 2009). Cette vision transformative (Booth, Nes \& Strømstad, 2003) se présente comme une caractéristique importante des initiatives visant à lutter contre l'exclusion. Elle se conçoit plus spécifiquement comme un processus de développement d'une culture, de politiques et de pratiques visant à soutenir l'apprentissage et la participation sociale de tous les élèves. De surcroît, elle se caractérise par la responsabilité partagée de chercher de nouvelles façons de construire les curriculums et de concevoir l'apprentissage afin que tous les élèves participent, s'acceptent et collaborent (Ainscow, 2009; Booth, Nes \& Strømstad, 2003; Brown \& Bauer, 2001). Il s'agit donc d'une approche collective et dynamique de résolution de problèmes visant à répondre positivement à la diversité des élèves (UNESCO, 2006) et à exploiter ces différences comme une ressource susceptible de contribuer à l'apprentissage. Cette conception n'est pas nouvelle selon différentes recensions d'écrits concernant la diversité en éducation (Corno \& Snow, 1986; Ducette, Sewell \& Poliner Shapiro, 1996; Prud'homme, Ramel \& Vienneau, 2011).

La vision transformative se distingue d'une vision assimilatrice de l'école (Booth et al., 2003). La gestion de la diversité dans une perspective assimilatrice s'associe plutôt à l'intégration scolaire (Moran, 2007) et se limite à placer ensemble tous les apprenants sans respecter et prendre en compte leurs intérêts, habiletés, expériences, genres, etc. L'intégration scolaire est une approche normative centrée sur l'intervention auprès des élèves ayant des besoins spéciaux. Elle cherche à amener ces derniers le plus près possible des pairs "normaux» par le recours à des services spécialisés et l'adaptation des programmes (Moran, 2007). Ainsi, l'école intégrative ne s'adapte et ne se transforme pas réellement (Mitchell, 2005); elle tente plutôt d'aider l'élève à s'adapter à une structure scolaire prédéfinie (Loreman, Deppeler \& Harvey, 2005).

Certaines critiques dénoncent la naïveté de l'approche inclusive ou encore présentent l'inclusion scolaire comme une utopie (Gillig, 2006). Effectivement, l'inclusion pourrait se concevoir comme la poursuite d'un but inatteignable. Cependant, de manière lucide, Booth et al. (2003) proposent de concevoir 
l'inclusion comme un mouvement et non comme une destination. Il s'agit de chercher à poser graduellement des actions concrètes, même petites, dans cette direction. La vision transformative influence la façon de se représenter l'ensemble des caractéristiques des écoles inclusives. En effet, elles se conçoivent alors comme des cibles vers lesquelles les acteurs éducatifs ont avantage à diriger leurs efforts concertés (Bergeron, en préparation).

\section{Quelques postulats d'une école inclusive}

Bien que l'inclusion scolaire puisse se déployer de manière variable selon les contextes, certains postulats et certaines caractéristiques semblent néanmoins faire un consensus au sein de la communauté scientifique.

Précisons d'entrée de jeu que l'école inclusive n'est pas tant préoccupée par ce qui distingue ou catégorise les apprenants et les apprenantes entre eux, mais plutôt par ce qui les rassemble. Ainsi, l'accent n'est pas posé sur les différences de rendement entre les élèves forts et les élèves moins forts ou encore entre les élèves handicapés et les élèves sans handicap, mais plutôt sur le potentiel, les forces cachées ainsi que la capacité de communiquer et d'apprendre de l'un et de l'autre (Hick, Kershner \& Farrell, 2009). Cette complémentarité des rôles est tout aussi à propos entres les différentes personnes qui composent l'équipeécole, le tissu familial et communautaire (Calderwood, 2000). En d'autres mots, l'école inclusive traduit un milieu où le projet éducatif ne se résume pas qu'au développement de connaissances et de compétences disciplinaires. Au contraire, son projet scolaire traduit le dépassement de soi et la mobilisation individuelle et collective. D'ailleurs, il y a plus de 15 ans déjà, Beninghof et Singer (19951996) identifiaient certaines croyances et intentions dirigeant les actions des éducateurs et des éducatrices en contexte d'inclusion, notamment: l'importance accordée au travail de collaboration entre éducateurs et éducatrices afin d'assurer une meilleure qualité de services aux élèves; les bénéfices de la présence d'autres élèves ayant des caractéristiques scolaires, raciales ou culturelles différentes; le souhait que l'environnement scolaire reflète le monde tel qu'il est à l'extérieur des murs de l'école; les bénéfices associés à l'accès à un curriculum flexible et à des compétences variées pour tous les élèves; le développement et le maintien d'un réseau naturel d'aide et de soutien (p. ex., cercle d'amis, adultes compréhensifs); le développement de relations d'entraide basées sur la richesse de chaque individu; l'absence de jugements et de prises de décisions fondées sur la crainte de l'échec plutôt que sur le développement professionnel; et le développement d'un sentiment de "pouvoir agir» chez tous les élèves et les éducateurs et éducatrices. Chose certaine, la recherche sur l'inclusion scolaire aura tôt fait de démontrer que pour adopter des "pratiques inclusives», il est nécessaire de dépasser son savoir d'action par la prise de conscience de ses propres cadres interprétatifs (Donnay \& Charlier, 2006; LaBoskey, 2007; Maroy, 2004; Rousseau 
\& Thibodeau, 2011; Whitehead, 1993) et que la confrontation des savoirs et de ces cadres interprétatifs contribue à cette reconstruction (Saussez \& Ewen, 2006; Wittorski, 2001).

\section{Caractéristiques des écoles inclusives et conditions de mise en cuvre}

Au cours des dernières années, certains travaux ont cherché à mettre en évidence les caractéristiques permettant de décrire les écoles inclusives (Rousseau \& Bélanger, 2004; Rousseau \& Prud'homme, 2010; Vienneau, 2002) et d'établir ses principales conditions de mise en œuvre. La présentation de ces caractéristiques ne vise pas à imposer un modèle unique d'inclusion scolaire, mais bien à préciser certains repères guidant les pratiques inclusives.

\section{Caractéristiques organisationnelles}

Trois caractéristiques d'ordre organisationnel ont été retenues: l'accès universel de l'école publique, condition sine qua non d'une école dite inclusive; le nécessaire leadership pédagogique exercé par la direction de l'école; et l'accessibilité aux ressources matérielles et humaines nécessaires à l'opérationnalisation du projet d'établissement (Booth \& Ainscow, 2005).

Tout d'abord, l'école inclusive accueille tous les élèves du quartier ou du village dans le groupe-classe de leur âge, et ce, indépendamment de leurs particularités (Mitchell, 2008; Vienneau, 2002). Elle est également celle qui se rend accessible sur le plan physique en mettant en place des dispositions d'accessibilité aux différents environnements physiques (Armstrong, 2006; Mitchell, 2008).

L'école inclusive repose également sur la présence d'un directeur ou d'une directrice ayant un leadership solide et offrant à son personnel les conditions pour soutenir la création d'une culture collaborative (Beattie, Jordan \& Algozzine, 2006; Booth et al., 2003; Mitchell, 2008; Rouse \& Florian, 1996; Rousseau \& Prud'homme, 2010; Shepherd \& Brody Hasazi, 2007). Cette caractéristique que Rouse et Florian (1996) associent aux écoles inclusives efficaces comprend des activités de formation continue, du temps pour planifier et évaluer les efforts, une flexibilité organisationnelle, un soutien à la motivation et des attentes élevées envers le personnel et les élèves. À ces caractéristiques s'ajoutent le rappel des valeurs de justice sociale, l'encouragement à l'utilisation de stratégies d'enseignement ayant fait l'objet de recherches, et l'animation de discussions pédagogiques qui mettent à profit les compétences et les savoirs des enseignants et des enseignantes (Shepherd \& Brody Hasazi, 2007).

Enfin, l'accessibilité à des ressources humaines et matérielles est une caractéristique indispensable des écoles inclusives (Mitchell, 2008). Qu'elles profitent aux élèves ou à l'enseignant ou l'enseignante, ces ressources sont le plus souvent déployées dans la classe. Ceci dit, certains élèves éprouvant des difficultés impor- 
tantes peuvent recevoir des services spécialisés en dehors de la classe lorsque cette forme de soutien est jugée nécessaire (Loreman, Deppeler \& Harvey, 2005). La coopération entre les élèves représente également une ressource importante à considérer (Rouse \& Florian, 1996; Rousseau \& Prud'homme, 2010). Soutien, support et formation continue représentent aussi des composantes essentielles de l'école inclusive dans la visée d'aider les acteurs scolaires à favoriser l'apprentissage de tous les élèves en classe ordinaire (Loreman et al., 2005; Rousseau \& Prud'homme, 2010).

\section{Caractéristiques pédagogiques}

La qualité de l'enseignement s'avère primordiale au sein d'une école inclusive efficace (Rouse \& Florian, 1996). En effet, l'inclusion scolaire implique différentes formes d'individualisation du processus enseignement/apprentissage par le recours à des stratégies d'enseignement et à des moyens d'évaluation variés et adaptés (Falvey \& Givner, 2005; Mitchell, 2005, 2008; Paré \& Trépanier, 2010; Roach, 2001; Vienneau, 2002). À ce titre, la différenciation pédagogique est considérée comme une composante importante de l'inclusion scolaire (AuCoin \& Vienneau, 2010; Paré \& Trépanier, 2010; Rousseau \& Prud'homme, 2010). Globalement, elle vise à soutenir la progression de tous les élèves par l'exploitation de situations d'enseignement/ apprentissage flexibles et variées favorisant la prise en compte des besoins de chacun. Leur mise en ouvre implique la création d'une communauté d'apprenants et d'apprenantes qui cherche à reconnaître, valoriser et exploiter la diversité (Prud'homme, en préparation).

Lorsque la différenciation pédagogique ne semble plus suffisante pour soutenir l'apprentissage de l'élève, l'individualisation du processus enseignement/apprentissage peut amener l'enseignante ou l'enseignante à faire usage d'accommodations ou de modifications (Beattie, Jordan \& Algozzine, 2006; Loreman et al., 2005; Paré \& Trépanier, 2010). Cette forme d'aménagement pédagogique ne date pas d'hier. En effet, dès 1978, Mary Warnock proposait dans son rapport, ce type d'interventions pour faciliter le déploiement de ceraines mesures d'enseignement spécialisé à l'intérieur des écoles publiques anglaises (special education in ordinarpy schools). Les travaux de Paré et Trépanier (2010) situent pour leur part l'accommodation et la modification comme des pratiques d'individualisation des apprentissages les plus souvent destinées aux élèves handicapés ou encore à ceux présentant des difficultés d'adaptation ou d'apprentissage importantes. L'accommodation, qui ne modifie pas le niveau de complexité d'une tâche ou du programme, vise à assurer l'accès aux apprentissages tout en permettant de répondre aux exigences du programme. L'utilisation d'une technologie d'aide à la lecture par l'élève qui éprouve des difficultés de lecture constitue, par exemple, une forme d'accommodation. La modification, quant à elle, est utilisée de manière exceptionnelle, lorsque la différenciation pédagogique et l'accommodation ne parviennent pas à assurer la progression de l'élève. Réduire la quantité de mots à écrire pour une production écrite constitue une forme de modifi- 
cation. Elle se présente donc comme une modification du programme ou des contenus d'apprentissage.

Toujours sur le plan pédagogique, nombreux sont les écrits scientifiques qui soulignent le potentiel de l'apprentissage coopératif pour soutenir l'inclusion scolaire (Bauer, 2001; Falvey \& Givner, 2005; Massé, 2010; Mitchell, 2008; Vienneau, 2002). Enfin, le recours aux grands principes de la pédagogie universelle (universal design for learning) est également associé aux pratiques inclusives (Bauer \& Matuszek, 2001; Bergeron, Rousseau \& Leclerc, 2011; Rousseau, 2010a).

\section{Caractéristiques sociales}

Certes, la qualité des relations sociales revêt une importance prépondérante dans l'école inclusive. En effet, on y préconise la participation de chaque élève à la vie sociale de la classe et de l'école ainsi qu'une participation optimale à toutes les activités d'apprentissage de son groupe-classe (Ainscow, 2009; Mitchell, 2005; Vienneau, 2002). Cette participation authentique s'appuie sur une reconnaissance et une valorisation de l'unicité de chaque apprenant et apprenante (Armstrong, 2006; Vienneau, 2004), ainsi que sur la création d'un climat de respect et d'acceptation de la différence (Hick, Kershner \& Farrel, 2009; Loreman \& al., 2005; Massé, 2010; Mitchell, 2008; Roach, 2001).

\section{Caractéristiques attitudinales}

Dans un autre ordre d'idées, l'école inclusive se caractérise par les efforts déployés autour du développement d'attitudes positives envers l'inclusion et envers les élèves handicapés ou présentant des difficultés d'adaptation ou d'apprentissage chez tous les membres du personnel scolaire. Les attitudes positives apparaissent comme une condition essentielle à l'amorce d'un projet d'inclusion (BlackHawkins, Florian \& Rouse, 2007; Rousseau, 2006) et au succès de l'inclusion (Loreman et al., 2005; Rousseau \& Prud'homme, 2010). Qui plus est, compte tenu du rôle que jouent les attitudes sur la mise en œuvre de pratiques inclusives, certains chercheurs et certaines chercheuses vont au-delà des attitudes des éducateurs et des éducatrices et misent plutôt sur le développement d'attitudes positives chez les décideurs, les décideuses et les gestionnaires de l'éducation (Peters, 2007).

\section{Conditions de mise en œuvre}

Outre les principales caractéristiques abordées plus haut, plusieurs écrits mettent en évidence certaines conditions contribuant à la mise en œuvre de pratiques plus inclusives (Pfeiffer \& Cundari, 1999; Praisner, 2003; Rousseau, 2006, 2007; Rousseau \& Bélanger, 2004; Rousseau \& Prud'homme, 2010; Schaffner \& Buswell, 1996; Shepherd \& Brody Hasazi, 2007; Vienneau, 2006). Parmi les conditions qui semblent faire un certain consensus, le développement d'une philosophie commune et d'un plan stratégique retiennent l'attention. En effet, 
puisque l'inclusion scolaire se donne pour défi de répondre aux besoins globaux de tous les élèves, tant au niveau scolaire, social et émotionnel qu'au niveau de la responsabilité personnelle et collective, il importe que tous les acteurs de l'éducation s'entendent sur les visées mêmes de leurs actions (philosophie commune) et sur les stratégies à mettre en place pour y parvenir (plan stratégique).

Intégré au projet éducatif de l'école, projet auquel tous seront appelés à participer (élèves, parents, personnel enseignant et non enseignant, administrateurs, administratrices, personnel de soutien et communauté), le plan stratégique devrait préciser les structures d'accueil et de soutien pour tous les élèves garantissant leur pleine participation à la classe et à l'école. Le plan devrait également préciser les objectifs poursuivis ainsi que les moyens et les ressources disponibles pour y parvenir. Ce plan devrait enfin prendre en considération certains facteurs facilitant l'inclusion scolaire, dont la réduction des ratios enseignant-enseignante/élèves, la restructuration de certains services, les structures favorisant la collaboration entre enseignants-enseignantes et l'ajout de ressources humaines et matérielles en fonction des besoins qui émergent. Sans surprise, le leadership de la direction d'école a un impact important sur l'efficacité de l'implémentation du plan stratégique visant le développement d'une école inclusive (Parent, 2010; Shepherd \& Brody Hasazi, 2007; Smith \& Smith, 2000).

La promotion de la diversité à l'école et dans la classe constitue une autre condition bien établie dans la littérature. Cette condition s'articule autour des «3 R», soit la reconnaissance des besoins de chacun, le respect de l'autre et de ses différences, et la responsabilité du bien-être de la personne (Shapiro, 1999). Dans cet esprit, promouvoir la diversité c'est également mettre un frein aux préjugés. À ce titre, tant les élèves que les acteurs de l'éducation gagnent à être sensibilisés au vocabulaire qui perpétue les préjugés. Les pratiques coopératives, plutôt que compétitives, permettent également de valoriser les différences (Schaffner \& Buswell, 1996). Enfin, tant la participation des élèves aux prises de décisions (Beaudoin, 2005a, 2005b) que les témoignages personnels (Salend, 2001) contribuent à la promotion de la diversité et au sentiment de bien-être de tous au sein de la communauté scolaire. Une fois de plus, le soutien de la direction d'école, par ses gestes et ses paroles, sert de modèle et favorise une atmosphère de soutien auprès de tous les acteurs de l'inclusion (Duchesne, 2010; Vienneau, 2010).

Par souci de favoriser la participation de tous les élèves aux activités scolaires et sociales de l'école, la création d'un comité visant la recherche de solutions pédagogiques innovatrices, voire l'adaptation de certaines activités, contribue au déploiement du projet inclusif (Schaffner \& Buswell, 1996). C'est par une approche de résolution de problèmes que les membres de ce comité mettront au point des stratégies et des activités favorisant la pleine participation de tous les élèves. Les mêmes auteurs encouragent également la création d'un comité visant à soutenir les enseignants et enseignantes à travers l'appropriation de pratiques plus inclusives. Plus récemment, Jorgensen, Schuh et Nisbet (2006) proposaient 
plutôt l'intégration au sein de l'école d'une personne facilitatrice de l'inclusion scolaire. Cette personne pourrait répondre aux besoins des enseignants, enseignantes et autres acteurs de l'école en soutenant la réflexion de ces derniers et en proposant des pistes d'actions en fonction des problèmes rencontrés. La personne facilitatrice peut également jouer un rôle dans la recherche de solutions visant la pleine participation scolaire et sociale de tous les élèves.

La flexibilité constitue une autre condition de mise en œuvre de l'école inclusive faisant consensus dans la littérature. Cette flexibilité renvoie à la prise de risque, à l'action innovante, à une approche de résolution de problèmes et à la collaboration entre l'ensemble des acteurs de l'école et de la commission scolaire ${ }^{2}$ (Bauer \& Matuszek, 2001). Non sans lien avec les pratiques pédagogiques, cette flexibilité contribue à l'émergence d'approches d'enseignement efficaces soutenues par des mesures de perfectionnement professionnel et de formation continue qui mettent à profit l'expertise des enseignants et enseignantes généralistes et des enseignants et enseignantes spécialisés (Morrocco, Walker \& Lewis, 2003; Shepherd \& Brody Hasazi, 2007).

\section{Les effets de l'inclusion scolaire sur le rendement scolaire}

À prime abord, il s'avère judicieux de questionner le concept même de rendement scolaire lorsque l'on s'intéresse aux effets de l'inclusion chez les élèves. Comme le rappellent si bien Black-Hawkins, Florian et Rouse (2007), «que prendre en considération dans l'étude du rendement scolaire, la dimension académique? sociale? émotionnelle? créative? physique?» (p. 3). Cette interrogation rejoint l'une des questions que Vienneau (2004) propose de se poser à l'issue de sa revue des articles et des recensions d'écrits consacrés à ce sujet et publiés pour l'essentiel au cours des années 1980 et 1990: de quelle inclusion parle-t-on au juste (puisqu'il existe une multitude de modèles d'inclusion scolaire)?; quel est ou quels sont les groupes d'élèves étudiés (les effets de l'inclusion pouvant évidemment varier selon les catégories de handicaps ou de difficultés présentes chez les élèves)?; les effets mesurés ou perçus (autre distinction importante) portent-ils sur l'apprentissage scolaire des élèves inclus (academic outcomes) ou sur leur développement social et les comportements affichés en classe (social and behavioral outcomes)? Enfin, quelle approche méthodologique a été privilégiée? La recherche quasi-expérimentale, l'étude de cas, le récit anecdotique?

En réponse à ces questions et à bien d'autres questions souvent soulevées ( $\mathrm{p}$. ex., quels sont les effets de l'inclusion scolaire chez les autres élèves de la classe ordinaire et quels sont ses impacts potentiels sur la qualité de l'enseignement offert au groupe-classe?), cette première recension des écrits amènera l'auteur à conclure que «'inclusion scolaire est réalisable sous certaines conditions minimales»; que «l'inclusion scolaire est souhaitable pour tous les élèves avec 
handicaps ou en difficulté»; enfin et surtout, que «l'inclusion scolaire en tant que modèle pédagogique a des effets positifs pour tous» (Vienneau, 2004, p. 147-148).

Dans une seconde «méta-recension des écrits», cette fois consacrée aux recensions publiées au cours de la décennie des années 2000 et couvrant la recherche menée tant en Amérique du Nord qu'en Europe, Vienneau (2010) présente les conclusions des six recensions suivantes: la recension de Lindsay (2007) portant sur les effets de l'inclusion scolaire chez l'ensemble des élèves avec handicaps ou en difficulté d'adaptation ou d'apprentissage (incluant à la fois les recherches dites comparatives et les recherches qualitatives); la recension de Ruijs et Peetsma (2009) portant sur les effets de l'inclusion scolaire chez les élèves en difficulté (effets sur l'apprentissage et effets sur le développement social); les recensions de Katz et Mirenda consacrées aux effets de l'inclusion sur l'apprentissage scolaire (2002a) et sur le développement social (2002b) des élèves inclus dans les écoles nord-américaines; la recension de Koster, Nakken, Pijl et Van Houten (2009) consacrée exclusivement à la dimension sociale de l'inclusion scolaire; enfin, la recension de Kalambouka, Farrell, Dyson et Kaplan (2007) portant sur les effets de l'inclusion scolaire chez les élèves de la classe ordinaire.

Dans sa recension, Lindsay (2007) présente, somme toute, un portrait relativement positif des effets de l'inclusion scolaire pour l'ensemble des élèves ayant des besoins particuliers (p. ex., aucune des recherches recensées n'affichait d'effets négatifs sur l'apprentissage contre 10 études classées comme ayant des effets positifs ou neutres). À la suite de leur recension consacrée aux effets de l'inclusion chez les élèves en difficulté (mild to moderate disabilities), Ruijs et Peetsma (2009) concluent pour leur part à des effets légèrement positifs sur l'apprentissage, mais expriment des positions plus réservées en ce qui concerne les effets d'ordre social et affectif. Quant aux recensions de Katz et Mirenda (2002a et 2002b), elles permettent d'une part de mettre en lumière certains effets positifs sur l'apprentissage, effets d'ordre davantage qualitatif que quantitatif (p. ex., la participation accrue des élèves inclus dans les activités d'apprentissage proposées au groupe-classe), et, d'autre part, d'illustrer le grand nombre et la très grande variété des effets associés à la dimension sociale de l'inclusion scolaire (p. ex., les manifestations d'amitié, la transformation des attitudes, l'adoption de nouvelles valeurs). La recension de Koster, Nakken, Pijl et Van Houten (2009) consacrée à la dimension sociale de l'inclusion scolaire aboutit à des conclusions similaires: «Les effets sociaux de l'inclusion scolaire se déclinent sous divers vocables (développement socioaffectif, acceptation sociale)»; ainsi, les résultats de la recherche dans ce domaine peuvent varier "suivant l'aspect de cette dimension sociale mis de l'avant par les chercheurs» (Vienneau, 2010, p. 253). Enfin, la recension de Kalambouka et al. (2007) portant sur les effets scolaires $(\mathrm{n}=50)$ et sociaux $(\mathrm{n}=21)$ de l'inclusion chez les élèves de la classe ordinaire cumule un total de 71 comparaisons, dont une grande majorité d'effets neutres $(58 \%)$ ou positifs $(23 \%)$, contre seulement sept effets négatifs $(9 \%)$. 
Une autre recension, soit celle de Norwich et Kelly (2005), fournit des résultats comparables aux recensions précédemment citées. Ainsi, la majorité des études recensées par ces auteurs traduisent une plus grande efficacité en matière d'apprentissage ou de rendement dans les classes ordinaires, ou aucune différence entre les classes ordinaires et les classes spéciales. Norwich et Kelly (2005) arrivent au même constat sur le degré d'assiduité ainsi que sur la nature et la qualité de la participation sociale des élèves en classe ordinaire. Toutefois, tout comme dans les recensions de Lindsay (2007) et de Ruijs et Peetsma (2009) analysées par Vienneau (2010), les effets d'ordre social et affectif sont plus mitigés, alors qu'une majorité d'études mettent en évidence une perception de soi plus négative chez les élèves handicapés ou en difficulté en classe ordinaire que chez ceux en classe spécialisée. Cela dit, les auteurs estiment que cette différence s'explique facilement par la comparaison sociale qui s'effectue à l'intérieur de tout groupe. En fait, ils en arrivent à la conclusion que dans les cas où la classe spéciale n'apporte aucun avantage de plus que la classe ordinaire, pourquoi la conserver?

\section{Conclusion}

Force nous est de constater que l'inclusion scolaire, qu'elle soit partielle ou totale, n'a pas toujours été accompagnée des transformations nécessaires pour assurer un maximum d'intégration pédagogique (participation de l'élève inclus aux activités d'apprentissage destinées à son groupe-classe) et une intégration sociale optimale de tous ces élèves. Le modèle pédagogique qui devrait accompagner l'inclusion, approche qu'on pourrait par ailleurs qualifier de pédagogie universelle (Bergeron, Rousseau \& Leclerc, 2011), est aujourd'hui désigné par un nombre sans cesse croissant de chercheurs et chercheuses, sous le vocable de "pédagogie de l'inclusion» ou de "pédagogie inclusive» (Rousseau, 2009). Si l'éducation inclusive constitue une notion polysémique par excellence (Bélanger $\&$ Duchesne, 2010) pouvant signifier «différentes choses pour les personnes qui en attendent différentes choses» (Fuchs \& Fuchs, 1994, p. 299, traduction libre), on s'entendra néanmoins sur une caractéristique commune à toutes les écoles plus ou moins inclusives: ce sont des établissements scolaires où l'on retrouve des équipes-écoles (Rousseau \& Thibodeau, 2011) ou des communautés d'apprentissage professionnelles (Leclerc \& Moreau, 2010) engagées dans un processus de changement.

De plus en plus d'écoles sont ainsi «en mouvement», image que Bélanger et Duchesne (2010) empruntent à deux chercheurs états-uniens pour désigner toutes ces écoles qui cherchent à adopter une pédagogie davantage "centrée sur l'enfant et adaptée à ses besoins plutôt que centrée sur des standards d'apprentissage et des rythmes d'apprentissage préétablis» (p. 5). En fait, on assiste depuis quelques années, en particulier au Canada francophone et en Europe, à un 
renouveau d'intérêt pour le projet d'une école inclusive et efficace, tel qu'en témoigne la publication récente de plusieurs collectifs ou numéros thématiques consacrés, par exemple, à la pédagogie de l'inclusion scolaire (Rousseau, 2010b; Rousseau \& Bélanger, 2004), aux transformations des pratiques éducatives nécessaires à l'inclusion scolaire (p. ex., Dionne \& Rousseau, 2006), aux modèles de services pouvant favoriser l'intégration ou l'inclusion scolaire (Trépanier \& Paré, 2010) et à la valorisation de la diversité en éducation (p. ex., Prud'homme, Ramel \& Vienneau, 2011).

Le «défi pédagogique» soulevé par l'inclusion scolaire et qui permet de distinguer la pédagogie de l'inclusion des seuls efforts de normalisation auxquels se limite trop souvent l'intégration scolaire (AuCoin, Goguen \& Vienneau, 2011) est l'élément-clé d'une véritable pédagogie de l'inclusion (Vienneau, 2005). Ceci dit, la pédagogie de l'inclusion, associée au modèle de l'inclusion totale (full inclusion), n'en est pas pour autant une pédagogie du «nivellement par le bas». Elle s'inscrit au contraire comme une composante intégrale des efforts de restructuration scolaire actuellement en cours qui font la promotion de normes de rendement élevées pour tous les élèves (Kendrick \& Labas, 2000).

En fait, il convient de signaler que de nombreuses études ont mis en lumière les liens opérationnels entre les écoles pratiquant une pédagogie de l'inclusion et les écoles dites efficaces (Arceneaux, 1994; Gallucci, 1997; Morefield, 2002; Slee $\&$ Weiner, 2001). Ainsi, la question à laquelle devrait désormais s'attaquer prioritairement les chercheurs et les chercheuses en inclusion scolaire est peut-être la suivante: quelles sont ces pratiques efficaces qui permettraient d'allier, certains diront réconcilier, l'intégration pédagogique optimale d'élèves avec handicaps et une pédagogie actualisante pour tous (Landry, Ferrer \& Vienneau, 2002), soit une pédagogie résolument mise au service du développement optimal du potentiel de chacun?

\section{Notes}

1 Cette section de l'article est inspirée de Bergeron, G. (en préparation). Soutenir un processus de développement de pratiques inclusives chez les enseignants du secondaire. Thèse de doctorat, Université du Québec à Trois-Rivières.

2 L'appellation «commission scolaire» désigne l'organisation scolaire locale.

\section{Références bibliographiques}

Ainscow, M. (2009). Developing inclusive education systems: What are the levers for change? In P. Hick \& G. Thomas (Ed.), Inclusion and diversity in education: Volume 2. Developing inclusive schools and school systems (pp. 1-13). London: SAGE.

Ainscow, M., Farrell, P. \& Tweddle, D. (2000). Developing policies for inclusive education: A study of the role of local education authorities. International Journal of Inclusive Education, 4 (3), 211-229.

Arceneaux, M. C. (1994). A Secondary School Experience: Is it inclusion or is it school reform? (ERIC Document Reproduction Service no. ED381 960). 
Armstrong, F. (2006). L'école inclusive: qu'est-ce que c'est et comment la construire? In R. M. Chevalier (Éd.), Actes du colloque international sous le thème: Pour une école inclusive... Quelle formation des enseignants? (pp. 73-81). Créteil: Services culture éditions ressources pour l'éducation nationale (SCÉRÉN) et centre régional de documentation pédagogique (CRDP) de l'académie de Créteil sous le thème.

AuCoin, A. \& Vienneau, R. (2010). Inclusion scolaire et dénormalisation. In N. Rousseau (Ed.), La pédagogie de l'inclusion scolaire: pistes d'action pour apprendre tous ensemble (pp. 63-86). Québec: Presses de l'Université du Québec.

AuCoin, A., Goguen, L. \& Vienneau, R. (2011). Pas plus spécial que nécessaire: analyse des politiques scolaires de la Nouvelle-Écosse à l'égard de l'inclusion scolaire des élèves avec handicaps. Éducation et francophonie, 39 (2), 23-49.

Bauer, A. M. (2001). Designing instruction in an inclusive classroom. In A. M. Bauer \& G. M. Brown (Éd.), Adolescent and inclusion, Transforming secondary school (pp. 85-108). Baltimore, MD: Brookes.

Bauer, A. M. \& Matuszek, K. (2001). Designing and evaluating accommodations and adaptations. In A. M. Bauer \& G. Myree Brown (Éd.), Adolescents and inclusion (pp. 139-166). Baltimore, MD: Brookes.

Beattie, J., Jordan, L. \& Algozzine, B. (2006). Making inclusion work: Effective practices for all teachers. Thousand Oaks, CA: Corwin Press.

Beaudoin, N. (2005a). Stepping outside your comfort zone. Larchmont, N.Y.: Eye On Education.

Beaudoin, N. (2005b). Elevating student voice. Larchmont, N.Y.: Eye On Education.

Beauregard, F. \& Trépanier, N. (2010). Le concept d'intégration scolaire... mais où donc se situe l'inclusion? In N. Trépanier \& M. Paré (Éd.), Des modèles de service pour favoriser l'intégration scolaire (pp. 31-56). Québec: Presses de l'Université du Québec.

Bélanger, S. (2010). Attitudes des différents acteurs scolaires à l'égard de l'inclusion. In N. Rousseau (Éd.), La pédagogie de l'inclusion scolaire (2e éd.) (pp. 111-132). Québec: Presses de l'Université du Québec.

Bélanger, N. \& Duchesne, H. (2010). Des écoles en mouvement: inclusion d'élèves en situation de handicap ou éprouvant des difficultés à l'école. In N. Bélanger \& H. Duchesne (Éd.), Des écoles en mouvement: inclusion d'élèves en situation de handicap ou éprouvant des difficultés à l'école (pp. 1-16) Québec: Presses de l'Université du Québec.

Beninghof, A. M. \& Singer, A.-L. F. (1995-1996). Ideas for inclusion: The school administrator's guide. Longmont, CO: Sopris West.

Benoit, V. \& Angelucci, V. (2011). Réflexions autour du concept de coenseignement en contexte inclusif. Éducation et francophonie, 39 (2), 105-121.

Bergeron, G. (en préparation). Soutenir un processus de développement de pratiques inclusives chez les enseignants du secondaire. Thèse de doctorat, Université du Québec à Trois-Rivières.

Bergeron, L., Rousseau, N. \& Leclerc, D. (2011). La pédagogie universelle: au coeur de la planification de l'inclusion scolaire. Éducation et francophonie, 39 (2), 87-104.

Black-Hawkins, K., Florian, L. \& Rouse, M. (2007). Achievement and inclusion in schools. New York: Routledge.

Bonvin, P. (2011). Développement d'un modèle d'évaluation d'un dispositif de soutien à l'inclusion scolaire. Education et francophonie, 39 (2), 250-271.

Booth, T. \& Ainscow, M. (2005). Guide de l'éducation inclusive. Québec: Institut québécois de la déficience intellectuelle (traduction).

Booth, T., Nes, K. \& Strømstad, M. (2003). Developing inclusive teacher education? Introducing the book. In T. Booth, K. Nes \& M. Strømstad (Éd.), Developing inclusive teacher education (pp. 1-14). London: RoutledgeFalmer.

Boutin, G. \& Bessette, L. (2009). Inclusion ou illusion? Élèves en difficulté en classe ordinaire: Défis, limites et modalités. Montréal: Éditions Nouvelles. 
Brown, G. D. \& Bauer, A. M. (2001). Qualities of an inclusive high school. In G. D. Bauer \& A. M. Brown (Éd.), Adolescent and inclusion, transforming secondary school (pp. 11-26). Baltimore, MD: Brookes.

Calderwood, P. (2000). Learning community: Finding common ground in difference. New York: Teachers College Press.

Corno, L. \& Snow, R. E. (1986). Adapting teaching to individual differences among learners. In M. C. Wittrock (Éd.), Handbook of research on teaching (3e éd.) (pp. 605-629). New York: MacMillan.

Dionne, C. \& Rousseau, N. (Éd.). (2006). Transformation des pratiques éducatives. Québec: Presses de l'Université du Québec.

Donnay, J. \& Charlier, B. (2006). Apprendre par l'analyse de pratiques: Initiation au compagnonnage. Namur: Presses universitaires de Namur. Sherbrooke: Éditions du CRP.

Doré, R., Wagner, S., Brunet, J.-P. \& Bélanger, N. (1998). L’intégration scolaire des élèves ayant une incapacité dans les provinces et territoires du Canada. Document téléaccessible à l'adresse www.cmec.ca/stats/pcera/compaper/98-44fr.pdf

Doré, R., Wagner, S., Brunet, J.-P. \& Bélanger, N. (1998). L'intégration scolaire des élèves ayant une incapacité dans les provinces et territoires du Canada. In Y. Lenoir, W. Hunter, D. Hodgkinson, P. de Broucker \& A. Dolbec (Éd.), Un programme pancanadien de recherche en éducation (pp. 233-260). Ottawa: Société canadienne pour l'étude en éducation (SCEE).

Ducette, J.-P., Sewell, T. E. \& Poliner Shapiro, J. (1996). Diversity in education: Problems and possibilities. In F. B. Murray (Éd.), The teacher educator's handbook (pp. 323-381). San Francisco: Jossey-Bass.

Ducharme, D. (2008). L'inclusion en classe ordinaire des élèves à besoins éducatifs particuliers. Montréal: Éditions Marcel Didier.

Duchesne, H. (2010). Initiatives inclusives en contexte manitobain: portrait de deux écoles. In N. Bélanger \& H. Duchesne (Éd.), Des écoles en mouvement (pp. 235-274). Ottawa: Presses de l'Université d'Ottawa.

Falvey, M. A. \& Givner, C. C. (2005). What is an inclusive school? In R. A. Villa \& J. S. Thousand (Éd.), Creating an inclusive school (2e éd., pp. 1-11). Alexandria, VA: Association for supervision and curriculum development.

Fuchs, D. \& Fuchs, L. S. (1994). Inclusive schools movement and the radicalization of special education reform. Exceptional Children, 60 (4), 294-309.

Gallucci, C. (1997). The MESH Manual for inclusive schools. Projet MESH: Making effective schools happen for all students. Washington Office of the State Superintendent of Public Instruction. Olympia (ERIC Document Reproduction Service no ED450 535).

Garbo, R. \& Albanese, O. (2006). L'intégration des élèves en difficulté et la formation des enseignants de soutien en Italie. In P.-A. Doudin \& L. Lafortune (Éd.), Intervenir auprès d'élèves ayant des besoins particuliers: quelle formation à l'enseignement? (pp. 31-44). Québec: Presses de l'Université du Québec.

Gillig, J.-M. (2006). Intégrer l'enfant handicapé à l'école (3e éd.). Paris: Dunod.

Haug, P. (2003). Qualifying teachers for the school for all. In T. Booth, K. Nes \& M. Strømstad (Éd.), Developing inclusive teacher education (pp. 97-115). London: RoutledgeFalmer.

Hick, P., Kershner, R. \& Farrell, P. T. (2009). Introduction. In P. Hick, R. Kershner \& P.T. Farrell (Éd.), Psychology for inclusion: New directions in theory and practice (pp. 1-10). Abingdon: Routledge.

Jorgensen, C. M., Schuh, M. C. \& Nisbet, J. (2006). The inclusion facilitator's guide. Baltimore, MD: Brookes.

Kalambouka, A., Farrell, P., Dyson, A. \& Kaplan, I. (2007). The impact of placing pupils with special educational needs in mainstream schools on the achievement of their peers. Educational Research, 49 (4), 365-382. 
Katz, J. \& Mirenda, P. (2002a). Including students with developmental disabilities in general education classrooms: educational benefits. International Journal of Special Education, 17 (2), 14-24.

Katz, J. \& Mirenda, P. (2002b). Including students with developmental disabilities in general education classrooms: social benefits. International Journal of Special Education, 17 (2), 25-35.

Kendrick, M. \& Labas, L. (2000). Building inclusive schools and pre-school communities, rural education and small schools (ERIC Document Reproduction Service no ED439 880).

Koster, M., Nakken, H., Pijl, S. J. \& Van Houten, E. (2009). Being part of the peer group: A literature study focusing on the social dimension of inclusion in education. International Journal of Inclusive Education, 13 (2), 117-140.

LaBoskey, V. K. (2007). The methodology of self-study and its theoretical underpinnings. In J. Loughran, M. Hamilton, V. Laboskey \& T. Russell (Éd.), International handbook of self study of teaching and teacher education practice (Tome 2, pp. 817-869). Dordrecht: Springer.

Lafortune, L. (2006). Vers une équité sociopédagogique: des élèves dans une collectivité. In P.-A. Doudin \& L. Lafortune (Éd.), Intervenir auprès d'élèves ayant des besoins particuliers: quelle formation à l'enseignement? (pp. 206-223). Québec: Presses de l'Université du Québec.

Landry, R., Ferrer, C. \& Vienneau, R. (rédacteurs invités) (2002). La pédagogie actualisante. Éducation et francophonie, 30 (2), 1-7. Sur Internet: http://www.acelf.ca/revue/30-2/

Leclerc, M. \& Moreau, A. C. (2010). Ensemble pour la réussite en littératie. Rapport 2010 sur les changements de pratiques en littératie et la réussite des élèves. Gatineau: Université du Québec en Outaouais.

Lindsay, G. (2007). Educational psychology and the effectiveness of inclusive education/ mainstreaming. British Journal of Educational Psychology, 77, 1-24.

Loreman, T., Deppeler, J. M. \& Harvey, D. H. (2005). Inclusive education: A practical guide to supporting diversity in the classroom. Crows Nest: Allen \& Unwin.

Maroy C. (2004). Sociologie des régulations de l'enseignement: une comparaison européenne. Recherches sociologiques, 35 (2).

Massé, L. (2010). Stratégie d'enseignement pour une classe inclusive. In N. Rousseau (Éd.), La pédagogie de l'inclusion scolaire, pistes d'action pour apprendre tous ensemble (pp. 351-379). Québec: Presses de l'Université du Québec.

Mitchell, D. (2005). Introduction. Sixteen propositions on the contexts of inclusive education. In D. Mitchell (Éd.), Contextualizing inclusive education: Evaluating old and new international perspective (pp. 1-21). Abingdon: Routledge.

Mitchell, D. (2008). What really works in special and inclusive education: Using evidence-based teaching strategies. Abingdon: Routledge.

Moran, A. (2007). Embracing inclusive teacher education. European Journal of Teacher Education, 3 (2), 119-134.

Moreau, A. C. (2010). Enseignante et enseignant inclusifs. In N. Rousseau (Éd.), La pédagogie de l'inclusion scolaire (2e éd., pp. 147-168). Québec: Presses de l'Université du Québec.

Morefield, J. (2002). Transforming education: recreating schools for all children. Consulté le 3 octobre 2009 dans www.newhorizons.org/article.morefield.html

Morroco, C. C., Walker, A. \& Lewis, L. R. (2003). Access to a schoolwide thinking curriculum: Leadership challenges and solutions. Journal of Special Education Leadership, 1 (1), 5-14.

Norwich, B. \& Kelly, N. (2005). Moderate learning difficulties and the future on inclusion. London and New York: RoutledgeFalmer.

Ouellet, S., Caya, I. \& Tremblay, M.-P. (2011). L'apport d'une communauté d'apprentissage pour développer des pratiques collaboratives et inclusives: une recherche-action. Éducation et francophonie, 39 (2), 207-226. 
Paré, M. \& Trépanier, N. (2010). Individualiser l'enseignement pour les élèves intégrés en classe ordinaire: mieux définir pour mieux intervenir. In N. Rousseau (Éd.), La pédagogie de l'inclusion scolaire (2e éd., pp. 287-306). Québec: Presses de l'Université du Québec.

Parent, G. (2010). Rôles de la direction dans une école inclusive. In N. Rousseau (Éd.), La pédagogie de l'inclusion scolaire (2e éd.) (pp. 169-194). Québec: Presses de l'Université du Québec.

Peters, S. (2007). Inclusion as a strategy for achieving education for all. In L. Florian (Éd.), The sage handbook of special education (pp. 116-130). London: Sage.

Pfeiffer, S. I. \& Cundari, L. (1999). Interagency collaboration: Recruffing obstacles and some possible solutions. In S. I. Pfeiffer \& L. A. Reddy (Éd.), Inclusion practices with special needs student: Theory, Research and application (pp. 125-144). Binghamton, N.Y.: Haworth Press.

Praisner, C. L. (2003). Attitudes of elementary school principals toward inclusion of students with disabilities. Exceptional Children, 69 (2), 135-145.

Prud'homme, L. (en préparation). La différenciation pédagogique: une perspective d'enseignement pouvant soutenir une société qui vise une saine démocratie. Montréal: CEC.

Prud'homme, L. (2010). Se former à un enseignement pour tous les élèves: un aller-retour théorie-pratique. In N. Rousseau (Éd.), La pédagogie de l'inclusion scolaire (2e éd., pp. 399-424). Québec: Presses de l'Université du Québec.

Prud'homme, L., Ramel, S. \& Vienneau, R. (2011). Valorisation de la diversité en éducation: défis contemporains et pistes d'action. Éducation et francophonie, 39 (2), 1-5.

Prud'homme, L., Vienneau, R., Ramel, S. \& Rousseau, N. (2011). La légitimité de la diversité en éducation: réflexion sur l'inclusion. Éducation et francophonie, 39 (2), 6-22.

Ramel, S. (2010). Quel impact de l'intégration et l'inclusion scolaires sur les enseignantes et enseignants? In N. Rousseau (Éd.), La pédagogie de l'inclusion scolaire (2e éd., pp. 383-398). Québec: Presses de l'Université du Québec.

Roach, V. (2001). Foreword. In C. M., Jorgensen (Éd.), Restructuring high schools for all students, taking inclusion to the next level (pp. $\mathrm{xv}-\mathrm{xx}$ ). Baltimore, $\mathrm{MD}$ : Brookes.

Rouse, M. \& Florian, L. (1996). Effective inclusive schools: a study in two countries. Cambridge Journal of Education, 26 (1), 71-85.

Rousseau, N. (2006). Développement d'une pratique pédagogique inclusive par une activité de recherche-action. Projet de recherche-action visant l'expérimentation d'interventions novatrices en collaboration avec le milieu de la recherche (Mesure 30054) - Bilan de recherche 2005-2006 [Rapport de recherche]. Trois-Rivières: Chaire de recherche NormandMaurice, Université du Québec à Trois-Rivières.

Rousseau, N. (2007). Développement d'une pratique pédagogique inclusive par une activité de recherche-action (volet écoles secondaires). Projet de recherche-action visant l'expérimentation d'interventions novatrices en collaboration avec le milieu de la recherche (Mesure 30054) Bilan de recherche 2006-2007 [Rapport de recherche]. Trois-Rivières: Chaire de recherche Normand-Maurice, Université du Québec à Trois-Rivières.

Rousseau, N. (2009). Conditions de mise en oeuvre d'une pédagogie inclusive. Le cas de la Gaspésie. Formation et pratiques d'enseignement en questions. Revue des HEP de Suisse romande et du Tessin, 9, 97-117.

Rousseau, N. (2010a). Vivement la pédagogie universelle pour les jeunes ayant des troubles d'apprentissage. In N. Rousseau (Éd.), La pédagogie de l'inclusion scolaire: pistes d'action pour apprendre tous ensemble (pp. 87-108). Québec: Presses de l'Université du Québec.

Rousseau, N. (Éd.). (2010b). La pédagogie de l'inclusion scolaire: pistes d'action pour apprendre tous ensemble. Québec: Presses de l'Université du Québec.

Rousseau, N. \& Bélanger, S. (2004). Dix conditions essentielles à la mise en place d'une école inclusive. In N. Rousseau \& S. Bélanger (Éd.), La pédagogie de l’inclusion scolaire (pp. 347-372). Québec: Presses de l'Université du Québec. 
Rousseau, N. \& Prud'homme, L. (2010). C'est mon école à moi aussi: dialogue sur les caractéristiques essentielles de l'école inclusive. In N. Rousseau (Éd.), La pédagogie de l'inclusion scolaire: pistes d'action pour apprendre tous ensemble (pp. 7-46). Québec: Presses de l'Université du Québec.

Rousseau, N. \& Thibodeau, S. (2011). S'approprier une pratique inclusive: regard sur le sentiment de compétence de trois équipes-écoles au cœur d'un processus de changement. Education et francophonie, 39 (2), 145-164.

Ruijs, N. M. \& Peetsma, T. T. D. (2009). Effects of inclusion on students with and without special educational needs reviewed. Science Direct. Consulté le 15 janvier 2010 dans http://www.sciencedirect.com

Salend, S. J. (2001). Creating inclusive classrooms: Effective and reflective practices. Colombus, $\mathrm{OH}$ : Merrill Prentice-Hall.

Saussez, F. \& Ewen, N. (2006). La démarche d'investigation critique, pierre angulaire d'une formation à des compétences professionnelles? D'un cadre conceptuel à l'expérimentation de projets de formation. Les cahiers scientifiques de l'ACFAS, 108, 45-67.

Schaffner, B. C. \& Buswell, B. E. (1996). Ten crucial elements for creating inclusive and effective school communities. In S. Stainback \& W. Stainback (Éd.), Inclusion: A Guide for Educators (pp. 49-66). Baltimore, MD: Brookes.

Shapiro, A. (1999). Everybody belongs. New York: RoutledgeFalmer.

Shepherd \& Brody Hasazi (2007). Leadership for social justice and inclusion. In L. Florian (Éd.), The sage handbook of special education (pp. 475-485). Thousand Oaks, CA: Sage.

Slee, R. \& Weiner, G. (2001). Education reform and reconstruction as a challenge to research genres: Reconsidering school effectiveness research and inclusive schooling. School Effectiveness and School Improvement, 12 (1), 83-98.

Smith, M. K. \& Smith, K. (2000). "I believe in inclusion, but... »: Regular education early childhood teachers' perceptions of successful inclusion. Journal of Research in Childhood Education, 14 (2), 161-180.

Stainback, W. \& Stainback, S. (1990). Support networks for inclusive schooling: Interdependent integrated education. Baltimore, MD: Brookes.

Thomazet, S. (2008). L'intégration a des limites, pas l'école inclusive! Revue des sciences de l'éducation, 34 (1), 123-139.

Trépanier, N. S. \& Paré, M. (2010). Des modèles de service pour favoriser l'intégration scolaire. Québec: Presses de l'Université du Québec.

UNESCO. (1994). The salamanca statement and framework for action on special needs education. Paris: UNESCO.

UNESCO. (2006). Principes directeurs pour l'inclusion: assurer l'accès à «l'éducation pour tous» . Paris: UNESCO.

Vienneau, R. (2002). Pédagogie de l'inclusion: fondements, définition, défis et perspectives. Education et francophonie, 30 (2), 257-286.

Vienneau, R. (2004). Impacts de l'inclusion scolaire sur l'apprentissage et le développement social. In N. Rousseau \& S. Bélanger (Éd.), La pédagogie de linclusion scolaire (pp. 125-152). Québec: Presses de l'Université du Québec.

Vienneau, R. (2005). Apprentissage et enseignement: théories et pratiques. Montréal: Gaëtan Morin Éditeur.

Vienneau, R. (2006). De l'intégration scolaire à une véritable pédagogie de l'inclusion. In C. Dionne \& N. Rousseau (Éd.), Transformation des pratiques éducatives (pp. 7-32). Québec: Presses de l'Université du Québec. 
Vienneau, R. (2010). Les effets de l'inclusion scolaire. Une recension des écrits (2000 à 2009). In N. Rousseau (Éd.), La pédagogie de l'inclusion scolaire (2e éd., pp. 237-263). Québec: Presses de l'Université du Québec.

Warnock, H. M. (1978). Special Educational Needs - Report of the Commitee of Enquiry into the Education of Handicapped Children and Young People (The Warnock Report). Rapport soumis au gouvernement britannique, Londres.

Whitehead, J. (1993). The growth of educational knowledge: Creating your own living educational theories. Bournemouth: Hyde Publications.

Wittorski R. (2001). Contribution de l'apprentissage expérientiel et de la science-action à la pratique professionnelle. In M.-P. MacKiewicz (Éd.), Praticien et chercheur-Parcours dans le champ social (pp. 107-118). Paris: L'Harmattan.

Mots-clés: Inclusion scolaire, intégration scolaire, diversité scolaire, pratiques efficaces

\section{Inklusion als Umgang mit Diversität: Von theoretischen Aspekten zu wirkungsvoller Unterrichtsgestaltung}

\section{Zusammenfassung}

Zahlreiche Länder wenden beträchtliche Anstrengungen auf, um ihre Bildungssysteme inklusiver zu gestalten. Dieses Vorgehen hat in den letzten Jahrzehnten mehrere Forschungsfelder geöffnet, darunter einerseits die Entwicklung von theoretischen Modellen zur inklusiven Schule und andererseits die Evaluation von verschiedenen mehr oder weniger inklusiven Unterrichtsmodellen. Der vorliegende Beitrag gibt einen aktuellen Überblick über diese beiden Forschungsfelder. Es werden zunächst verschiedene konzeptionelle Aspekte wie Definitionen, Postulate für eine inklusive Schule, Charakteristiken einer inklusiven Pädagogik und wesentliche Bedingungen für die Umsetzung dargestellt. Der nächste Abschnitt beschäftigt sich mit den Ergebnissen der Inklusion hinsichtlich der schulischen Leistungen und der sozialen Entwicklung der Schülerinnen und Schüler. Zum Abschluss werden mit dem dritten Forschungsfeld wirksame Formen der Unterrichtsgestaltung beschrieben.

Schlagworte: Schulische Inklusion, schulische Integration, Diversität, wirksamer Unterricht 


\section{L'inclusione scolastica per gestire la diversità: dagli aspetti teorici alle cosiddette pratiche efficaci}

\section{Riassunto}

Moti paesi investono molto per sviluppare sistemi scolastici più inclusivi. Questi sforzi hanno portato, negli ultimi anni, all'identificazione di diverse direzioni di ricerca, ad esempio lo sviluppo di modelli teorici di inclusione scolastica, così come alla valutazione dell'impatto di pratiche più o meno inclusive. Questo contributo cerca di fare sintesi del lavoro svolto in queste due direzioni. Vengono presentati diversi aspetti concettuali: definizioni, postulati relativi alla scuola inclusiva, caratteristiche dell'educazione inclusiva e le principali condizioni di realizzazione. Gli effettivi dell'inclusione scolastica sull riuscita degli allievi viene presentata nell'ultima parte dell'articolo, prima di concludere con una recente ricerca dedicata alle buone pratiche.

Parole chiave: Inclusione scolastica, integrazione scolastica, diversità scolastica, pratiche efficaci

\section{School inclusion to manage diversity: From theoretical aspects to best practices}

\section{Abstract}

Several countries unfold considerable efforts in order to develop more inclusive school systems. This move generated different research directions over the past decades, such as the development of theoretical models of school inclusion, as well as the assessment of more or less inclusive practices effects. This contribution intends to synthesize work carried out in each of these two directions. Different conceptual aspects will be presented: definitions, inclusive school assumptions, inclusive education features, and main implementation conditions. The effects of school inclusion on students' learning outcome and social development will be presented in the last section, before concluding with recent research related to best practices.

Key words: Inclusive school, school integration-mainstreaming, school diversity, best practices 